\title{
Toma de decisiones basada en la productividad en Pymes manufactureras: aproximación desde la Lógica Difusa *
}

\author{
Productivity-Based Decision Making in Manufacturing SMEs: A Fuzzy Logic \\ Approach
}

\author{
Julio Albeiro Londoño-Patiño \\ Magister en Gestión de la Innovación Tecnológica, Cooperación \\ y Desarrollo Regional, Servicio Nacional de Aprendizaje - SENA, \\ Medellín-Colombia, jlondonop@hotmail.com
}

Cómo citar / How to cite

Londoño-Patiño, J. A. (2020). Toma de decisiones basada en la productividad en Pymes manufactureras: aproximación desde la Lógica Difusa. Revista CEA, v. 6, n. 12, 181-207.

https://doi.org/10.22430/24223182.1507

Recibido: 20 de marzo de 2020

Aceptado: 4 de mayo de 2020

\begin{abstract}
Resumen
Uno de los retos para la pequeña y mediana empresa (PYME) es entender y conocer en qué momento debe hacer los cambios organizacionales y tomar las decisiones adecuadas que no afecten su nivel de productividad. Comprender los factores que involucran la toma de decisiones en las Pymes está relacionado con la necesidad de entender el entorno de los negocios y sus variables de productividad laboral referido a la efectividad de las buenas prácticas de productividad operacional (BPPO), sus sistemas de apoyo y de transferencia de conocimiento aplicado a la administración de la producción. Para entenderlo, se debe analizar el contexto desde una perspectiva integral. Es por esta razón que el presente artículo pretende examinar la toma de decisiones basada en la productividad en Pymes manufactureras a través de la utilización de lógica difusa, tomando como caso 6 Pymes de Medellín, Colombia, donde se empleó el modelo Índice de Productividad Total de Factores (IPTF). Con respecto a la metodología, se hace desde la ejecución rigurosa de los diferentes pasos del método borroso en las Pymes con un enfoque descriptivo, lo que permitió seleccionar las condiciones más cercanas a los principios difusos, confirmando la efectividad de la lógica difusa en el proceso de toma de decisiones empresariales. Además, como resultado significativo, se aporta a líneas de investigación, con una orientación metodológica basada en la productividad (BPPO) que apoyan la toma de decisiones empresariales.
\end{abstract}

\footnotetext{
* Este artículo se deriva del proyecto titulado «Toma de decisiones basada en la productividad en Pymes manufactureras:
} aproximación desde la Lógica Difusa» y ha sido financiado con recursos propios. 
Palabras clave: lógica difusa, Pymes manufactureras, competitividad, toma de decisiones.

Clasificación JEL: D81; L69.

\begin{abstract}
One of the challenges that small- and medium-sized enterprises (SMEs) face is knowing when to make the necessary organizational changes and the appropriate decisions that will not affect their level of productivity. Identifying the factors involved in decision making in SMEs is related with the need to be aware of their business environment and labor productivity variables in terms of effectiveness of Best Operational Productivity Practices (abbreviated BPPO in Spanish), support systems, and knowledge transfer applied to production management. For this purpose, the context must be analyzed from a comprehensive perspective. Therefore, the aim of this study is to examine productivity-based decision making in manufacturing SMEs using the fuzzy logic approach. As a sample, we analyze six SMEs in the city of Medellín, Colombia, by means of the Total Factor Productivity (TFP) model. Regarding the methodology of this study, we rigorously applied the different steps of the fuzzy method to the SMEs under study following a descriptive approach. This allowed us to select the conditions that were closer to the fuzzy principles, thus confirming the effectiveness of fuzzy logic in business decision making. In addition, our findings contribute to lines of research by providing a methodological framework focused on productivity (BOPP) which can support business decision making.
\end{abstract}

Keywords: Fuzzy logic, small - and medium - sized enterprises (SMEs), competitiveness, decision making.

JEL classification: D81; L69.

\title{
1. INTRODUCCIÓN
}

Las Pymes manufactureras están inmersas en cambios derivados por el entorno globalizado, los cuales exigen incesantes esfuerzos por incrementar la calidad y la productividad, además de reducir los costos y los tiempos de entrega a fin de lograr posiciones ventajosas en el mercado (Pérez Rave et al., 2011; Maldonado-Pinto \& Portilla-Barco, 2020; Larsson et al., 2017). Para esto, las organizaciones se ven obligadas a mejorar su nivel de competitividad tomando decisiones que involucren temas asociados a la productividad y no solamente a inversiones de adopción tecnológica que representan cambios radicales administrativos y son aislados de las decisiones estratégicas operativas (Cassou \& De Oliveira, 2011). En lo que respecta a lo que está sucediendo en el entorno de los negocios, resulta de gran importancia encontrar instrumentos y metodologías en la toma de decisiones en las que se incluya la productividad de las empresas, recursos tecnológicos, desarrollo de capacidades, entre otros (Özemre \& Kabadurmus, 2020).

Para la PYME la toma de decisiones es indispensable, puesto que una decisión mal tomada puede conllevar una situación comprometedora (Brunetto \& Farr-Wharton, 2007). La idea es tener un claro entendimiento del entorno de actuación, es decir, que la toma de decisión contribuya a la adopción de mejoras de la organización (Khan et al., 2019). Una vez adecuada, esta decisión permite tener claro los pasos a seguir para lograr el éxito empresarial; sin embargo, uno de los principales 
problemas en las Pymes es la falta de capacidades gerenciales de los puestos estratégicos. Así, «las personas encargadas de la toma de decisiones deben estar capacitadas y conocer todas las características y pasos que constituyen este proceso, con miras a eliminar, en lo posible, valoraciones subjetivas al momento de elegir un curso de acción» (Cabeza de Vergara \& Muñoz Santiago, 2010). Las organizaciones deben apoyarse en herramientas que proporcionen información y datos que aseguren una de toma de decisiones correctamente procesada y analizada para el mantenimiento de su sostenibilidad en el tiempo.

Conocido es que, en la actualidad, varias de las «decisiones en el marco de las organizaciones se toman sin considerar explícitamente las etapas de ese proceso o los métodos cuantitativos y cualitativos existentes en las distintas ramas» (Díaz Duarte, 2005). A partir de la necesidad expuesta, el presente trabajo se enfoca en la utilización de la lógica difusa para la toma de decisiones. Se realizó una revisión de la literatura especializada en este campo y su aplicación bajo el contexto de 6 Pymes manufactureras de la ciudad de Medellín. Se identifica que la lógica difusa puede ofrecer al proceso de toma de decisiones empresariales certidumbre, en particular, en lo relativo a la administración de los negocios. Lo anterior como consecuencia que los resultados de las intervenciones arrojan múltiples criterios en la asignación de valor.

El documento tiene seis partes. La primera se dedica a una revisión de la literatura con el fin de contextualizar al lector en la toma de decisiones en las Pymes, además de la justificación del análisis y los resultados esperados. De igual forma, se presenta el enfoque basado en procesos y el marco metodológico. En la segunda se da un acercamiento a los términos y principios de la lógica difusa junto a las modelaciones con sus diferentes variables que componen una alternativa útil para el propósito planteado. La tercera hace un enfoque metodológico descriptivo utilizando como métrica fundamental el índice de productividad total de factores (IPTF), que agrupa las variables fundamentales que requiere una organización para medir su productividad real, tanto el problema de decisión como los criterios decisores. La cuarta parte hace referencia a los resultados fundamentales del trabajo. Finalmente, el quinto presenta unas líneas de discusión, y la sexta parte cierra con las conclusiones.

\section{MARCO TEÓRICO}

\section{Lógica difusa (fuzzy logic)}

Rivas (2009), la lógica analiza el razonamiento en todos sus métodos y principios posibles. La lógica clásica trata las proposiciones que pueden ser verdaderas o falsas. Las primeras lógicas evaluadas fueron propuestas por Lukasiewicz en 1930 como extensión de su lógica trievaliada (verdadero, falso e indeterminado) (García-Alegre, 1991; Nagy et al., 2019). Es importante comprender la relación existente entre la forma de razonar y el medio que nos rodea (entorno).

Lo anterior se debe a que los procesos presentan cierto grado de vaguedad, incertidumbre o probabilidad que pueden producir nuevas respuestas sobre las mismas acciones, lo que afirma la capacidad de adaptación que tienen las empresas. En general, las organizaciones aprenden que no todo es blanco o negro, sino que hay diferentes tipos de tonalidades de grises; este entendimiento a su vez depende de la forma en que son percibidas las realidades (Strefezza, 2009). 
Generalmente:

cualquier problema del mundo puede resolverse como un conjunto de variables de entrada (espacio de entrada) y obtener un valor adecuado de variables de salida (espacio de salida)., la lógica difusa permite establecer este marco de una forma adecuada, atendiendo a criterios de significado (y no de precisión). (Morcillo Loro, 2017, p. 7)

Strefezza (2009), la lógica difusa fue planteada por Zadeh en 1965, donde publicó su trabajo Fuzzy Sets en 1965, y Fuzzy Algorithms en 1968, textos que detallan los fundamentos matemáticos relacionados a la teoría de conjuntos difusos y a la teoría de lógica difusa. Zadeh expresa la forma de representar la vaguedad matemáticamente y su asociación a los eventos de la vida diaria y constituye una generalización de la lógica clásica, que es determinista: verdadero o falso; variación entre cero y uno Strefezza (2009). De acuerdo al mencionado autor, un conjunto difuso posee un grado de membresía determinada por una clase de objetos con un continuum. Por lo cual, la lógica difusa, puede manejar el razonamiento aproximado.

Por esta razón, las variables lingüísticas se utilizan en la lógica difusa. Así, por ejemplo, una variable lingüística como la satisfacción del cliente, competitividad, productividad, entre otras, puede tener valores como el de insolvencia y solvencia empresarial. El valor solvencia permite categorizar a los elementos del universo de competitividad con mayor detalle, pues se les dan valores que varían en pertenencia dentro del conjunto (Zadeh, 1965).

Una de las consideraciones que termina siendo una ventaja importante es la transición de pertenencia de un conjunto a otro. Bajo la mirada empresarial del desempeño, la variable de competitividad y productividad es considerada como dos conjuntos: uno A, insolvencia, y otro B, solvencia. Cada uno, tendrá una función que define su membresía del grupo y que será denominada como grado de proximidad ( $x$ ), en la cual x representa el grado de membresía del conjunto. De esta forma, entre más cerca esté el valor de $x$ de uno (1), mayor será la membresía de dicho conjunto (Ruvalcaba \& Vermonden, 2015).

En consecuencia, las tres operaciones básicas entre conjuntos, unión, intersección y complemento, se generalizan en los conjuntos difusos. Sin embargo, la definición de las operaciones está dadas en función de pertenencia al conjunto [0; 1], estas combinaciones difusas se comportan de igual modo que las combinaciones en conjuntos reales (González, 2007). Estas operaciones se pueden apreciar en la Figura 1. 


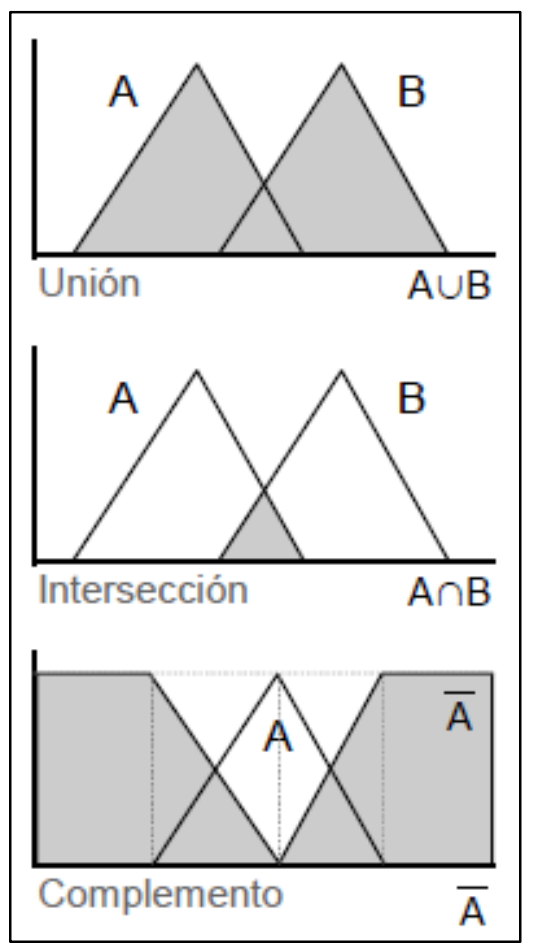

Figura 1. Descripción gráfica de operaciones estándar con conjuntos difusos.

Figure 1. Graphical description of standard operations with fuzzy sets Fuente: (Morcillo Loro, 2017).

Como lo expresa Lizarzaburu (2014), la información es uno de los recursos más valiosos para las empresas (Chen, 2010), por lo que es importante saber cómo utilizarla adecuadamente, en particular, en la toma de decisiones. Es importante, en un contexto organizacional, desarrollar y hacer uso de información confiable, relevante y útil (Godlee et al., 2004). Estas características son fundamentales, especialmente en la información financiera, que es vital para los procesos de toma de decisiones de los actores económicos (Lizarzaburu, 2014).

Bajo el contexto de la toma de decisiones empresariales, surgen los modelos de predicción como una respuesta aproximada a esta necesidad. Como lo expresa Belalcázar \& Trujillo (2016) uno de ellos es el Modelo Z de Altman, desarrollado por Edward Altman (1968), cuya propuesta identifica las relaciones lineales de los indicadores claves (insolvencia y solvencia) de las compañías para clasificarlas según su probabilidad de impago (Altman, 1968). Para esto, se determina cuáles indicadores son los más importantes para detectar un potencial incumplimiento, cuales valores debe asignársele a los indicadores seleccionados y por último cómo se establecen objetivamente estas ponderaciones (Ortega et al., 2010).

\section{Lógica difusa y modelaciones}

La modelación en la lógica difusa enmarca un multicriterio estructural que combinado con la incidencia de los conjuntos difusos representa los flujos de información y de los procesos a través de los cuales las organizaciones en el desarrollo de sus estrategias «se conectan a los estados del sistema con los puntos de decisión, y también con las consecuencias de esas decisiones, mostrando ciclos de 
realimentación de refuerzo o balance» (Martínez Rojas \& Andrade Sosa, 2016, p. 150) de acuerdo a los indicadores del desempeño empresarial.

Relaciones estructurales y funcionales que como lo expresa Arango Serna et al., (2012) citando a Goncalves (2008) quien interpreta y analiza el desempeño estratégico a través de dos indicadores mediante la lógica difusa, donde para evaluar el desempeño convierte la visión y la estrategia en objetivos medibles. Valenzuela (2008) por su parte utilizó un factor difuso en el sistema de gestión de indicadores para el mejoramiento continuo de los procesos empresariales.

Bajo este contexto los otros autores Mallo et al., (2006) expresan que la incidencia difusa aplicada en matrices permite validar las relaciones entre los diferentes factores críticos de éxito. Además, las matrices de incidencias validan herramientas de mando de control empresarial como el BSC en su relación con los indicadores. En esta misma línea, Lüttich y Fiege (2005) demostraron la importancia de los de los conjuntos difusos en el contexto del cuadro de mando integral para las organizaciones.

Sin embargo, continua Arango Serna et al., (2012) que la investigación de Bobillo et al., (2009) demuestran el éxito que tienen la lógica difusa en el tratamiento del BSC. Para Mallo et al., (2006) la cuantificación de los factores críticos de éxito toma criterios de certidumbre con la aplicación de la lógica difusa. De igual forma, la relevancia de la lógica difusa para la interpretación de los criterios de valor permitió realizar operaciones y mediciones de variables cualitativas. Así, de esta manera, la lógica difusa permite operar con factores de origen diverso y facilita la cuantificación y cualificación de las variables. Por último, Arango Serna et al., (2012) expresa que la inferencia difusa sirve de base para que el análisis de los resultados incluya criterios de incertidumbre y certidumbre presentes en cualquier sistema de gestión empresarial. A continuación, en la Tabla 1 se relacionan modelos desarrollados por diferentes autores.

Tabla 1. Modelación en toma de decisiones

Table 1. Decision-making models

\begin{tabular}{|c|c|c|c|}
\hline \multicolumn{4}{|c|}{ Investigaciones previas (métodos, resultados, conclusiones) } \\
\hline Modelo & Año & Autor (es) & Orientación / Elementos y conceptos presentes \\
\hline $\begin{array}{l}\text { Modelo de los } \\
\text { procesos de } \\
\text { decisión }\end{array}$ & 1955 & Simón & $\begin{array}{l}\text { La toma de decisiones organizacionales. } \\
\text { Procesos de toma de decisiones } \\
\text { Información }\end{array}$ \\
\hline Modelo racional & $\begin{array}{l}1992- \\
1963\end{array}$ & $\begin{array}{c}\text { March y } \\
\text { Cyert }\end{array}$ & $\begin{array}{l}\text { La toma de decisiones racional en las organizaciones. } \\
\text { Racionalidad limitada } \\
\text { Información } \\
\text { Certidumbre e incertidumbre } \\
\text { Estrategias y simplificaciones para la toma de decisiones } \\
\text { organizacionales }\end{array}$ \\
\hline Modelo político & 1971 & Allison & $\begin{array}{l}\text { La toma de decisiones como escenario en el que interviene la } \\
\text { política como juego de decisiones. } \\
\text { Factores y fuentes de persuasión, regateo y negociación } \\
\text { Información }\end{array}$ \\
\hline $\begin{array}{l}\text { Modelo Can } \\
\text { Garbage }\end{array}$ & 1972 & $\begin{array}{l}\text { Cohen, } \\
\text { March y } \\
\text { Olsen }\end{array}$ & $\begin{array}{l}\text { La toma de decisiones estratégicas. } \\
\text { Organizaciones como anarquías organizadas } \\
\text { Información }\end{array}$ \\
\hline
\end{tabular}




\begin{tabular}{|c|c|c|c|}
\hline $\begin{array}{l}\text { Modelo de proceso } \\
\text { de toma de } \\
\text { decisiones } \\
\text { estratégica }\end{array}$ & 1976 & $\begin{array}{l}\text { Mintzberg, } \\
\text { Raisinghani y } \\
\text { Theoret }\end{array}$ & $\begin{array}{l}\text { La toma de decisiones estratégicas en una organización. } \\
\text { Procesos o fases de la toma de decisiones } \\
\text { Información }\end{array}$ \\
\hline $\begin{array}{l}\text { Modelo jerárquico } \\
\text { de toma de } \\
\text { decisiones } \\
\text { participativa }\end{array}$ & 1992 & $\begin{array}{l}\text { Evans y } \\
\text { Fischer }\end{array}$ & $\begin{array}{l}\text { La toma de decisiones participativa mediando entre la autonomía } \\
\text { del desempeño de los individuos y el control percibido del trabajo } \\
\text { que realizan en las organizaciones. } \\
\text { Toma de decisiones colectivas } \\
\text { Percepción de control } \\
\text { Autonomía el trabajo }\end{array}$ \\
\hline
\end{tabular}

Modelo colectivo
$\begin{aligned} & \text { de toma de } \\ & \text { decisiones }\end{aligned}$

La toma de decisiones colectiva en la negociación en Netherlands.

Modelo de cambio

Modelo de conflicto

Procesos de negociación

Sistema de base de datos

Simulación y producción de alternativas de cambio

\begin{tabular}{|c|c|c|c|}
\hline $\begin{array}{l}\text { Modelo de } \\
\text { decisiones médicas }\end{array}$ & 2003 & Whitney & $\begin{array}{l}\text { La toma de decisiones médicas como un proceso } \\
\text { compartido/colectivo. } \\
\text { Certeza en la toma de decisiones medicas } \\
\text { Factores objetivos y subjetivos que intervienen en el } \\
\text { proceso }\end{array}$ \\
\hline $\begin{array}{l}\text { Modelo de toma } \\
\text { de decisión }\end{array}$ & 2003 & $\begin{array}{l}\text { Balali, } \\
\text { Badredin, } \\
\text { Reza y } \\
\text { Farhangi }\end{array}$ & $\begin{array}{l}\text { La toma de decisiones de gerentes iraníes y su comportamiento. } \\
\text { Percepción y emoción } \\
\text { Comportamiento, actitud, personalidad y valores } \\
\text { Experiencia y conocimiento }\end{array}$ \\
\hline $\begin{array}{l}\text { Modelo ceo-asesor } \\
\text { para la toma de } \\
\text { decisiones } \\
\text { estratégicas }\end{array}$ & 2005 & $\begin{array}{l}\text { Arendt, } \\
\text { Priem y } \\
\text { Achidi }\end{array}$ & $\begin{array}{l}\text { La toma de decisiones estratégicas reconocimiento el rol de un ceo } \\
\text { a altos niveles de dirección y el asesoramiento de equipos de } \\
\text { gerentes que toman las decisiones. } \\
\text { Toma de decisiones estratégicas } \\
\text { ceo } \\
\text { Equipos de gerentes } \\
\text { Información }\end{array}$ \\
\hline $\begin{array}{l}\text { Modelo dinámico } \\
\text { prospectivo de } \\
\text { toma de decisiones }\end{array}$ & 2005 & Kanner & $\begin{array}{l}\text { La toma de decisiones prospectiva y sus salidas (decisiones) a partir } \\
\text { de la percepción del contexto organizacional. } \\
\text { Percepción del contexto } \\
\text { Procesos de decisión } \\
\text { Factores de riesgo } \\
\text { Información }\end{array}$ \\
\hline $\begin{array}{l}\text { Aplicación de } \\
\text { Modelo de } \\
\text { despliegue } \\
\text { funcional en la } \\
\text { toma de decisiones }\end{array}$ & 2006 & $\begin{array}{l}\text { Yung, Ko, } \\
\text { Kwan, Tam, } \\
\text { Lam, Ng y } \\
\quad \text { Lau }\end{array}$ & $\begin{array}{l}\text { La toma de decisiones orientada al desarrollo de nuevos productos } \\
\text { organizacionales a partir del Modelo de desarrollo funcional (fdm). } \\
\text { Despliegue funcional de calidad (qfd) } \\
\text { Requerimientos y atributos funcionales para el desarrollo } \\
\text { de productos } \\
\text { Línea de programación y optimización de recursos } \\
\text { Solución de problemas }\end{array}$ \\
\hline $\begin{array}{l}\text { Modelo de toma } \\
\text { de decisiones en la } \\
\text { cooperativa }\end{array}$ & 2007 & López & $\begin{array}{l}\text { La toma de decisiones estratégicas de una cooperativa. } \\
\text { Procesos o etapas para la toma de decisiones estratégicas }\end{array}$ \\
\hline
\end{tabular}


Ambiente interno y externo organizacional para la toma de decisiones

La toma de decisiones económicas a partir de un conjunto de heurísticas racionales de búsqueda, análisis y selección de

Modelo heurístico racional de toma de decisiones $2010 \quad$ Grandori alternativas.

económicas

Centrado en las decisiones económicas y sus características particulares.

Heurísticas racionales para la toma de decisiones económicas

Racionalidad

Modelo de proceso

de toma de

$2011 \quad$ Citroen

Las fases racionales a través de las que se toman decisiones. Se

decisiones enfatiza en el uso de información asociada a:

- Información interna (ambiente interno)

- Información externa (ambiente externo)

Fuente: (Rodgríguez Cruz, 2014, p. 90)

\section{Toma de decisiones con incertidumbre}

Cabeza de Vergara y Muñoz (2010) analizan cómo se desarrolla la toma de decisiones, para lo cual presentan «una breve explicación de cada uno de los modelos utilizados en las organizaciones. En esta línea exponen los resultados obtenidos a una muestra de empresas Pymes de la ciudad de Barranquilla», en cuyos resultados hay una alta tendencia para resolver problemas utilizar la intuición y la experiencia del tomador de decisiones. De esta manera, Cabeza de Vergara y Muñoz (2010) expresan que se puede apreciar la necesidad de modelos de simulación que «permita tener más certeza a la hora de tomar un curso de acción ante una situación problemática». Por último, plantean Cabeza de Vergara y Muñoz (2010) que «la toma de decisiones es indispensable para las empresas pues una decisión mal tomada, puede conllevar a una situación comprometedora».

De acuerdo al trabajo realizado por Castiblanco Ruiz (2013), la relación de la lógica difusa con la toma de decisiones, especificando las condiciones de incertidumbre, conducen a un análisis de interacciones de la lógica difusa con los criterios de decisión y sus relaciones existentes con la incertidumbre conllevan a un resultado más objetivo referido a la toma de decisión deseada.

Igualmente, se han desarrollado diferentes escritos «sobre los cambios en los paradigmas organizacionales y cómo estos pueden influir en los métodos de ingeniería que se utilizan en la toma de decisiones» (Prince \& Vera, 2005). Asu vez, expresan que los métodos clásicos utilizados en la toma de decisiones, se pueden dividir en dos: los modelos deductivos y los modelos inductivos. Sin embargo, ambos modelos no pueden desprender la indeterminabilidad para el tomador de decisiones en el quehacer empresarial (Prince \& Vera, 2005).

Bajo este contexto, continua Prince \& Vera (2005) que la incertidumbre, lleva consigo la inestabilidad, lo complejo, y lo difuso. Por lo cual, la adaptabilidad de lo confuso está dada por la posibilidad de utilizar métodos que combinen lo cualitativo junto a los cuantitativo con el fin de aumentar la certidumbre en la toma de decisiones. Finalmente, para Prince \& Vera (2005) la toma de decisiones empresariales alineadas a la visión estratégica no escapa al orden cualitativo, debido a los cambios 
constantes del entorno; mientras que las de orden operacional en sus interacciones propias suelen ser de tipo cuantitativo.

Frente a la modelación en productividad, citando a Medina Fernández de Soto (2009) se han desarrollado modelos de medición de la productividad, entre ellos: la productividad parcial, la total (Summanth, 1990) y la de valor agregado. Sin embargo, estos modelos no explican las relaciones entre la estrategia empresarial y la productividad (Medina Fernández de Soto, 2009).

El modelo integral de productividad expuesto por Medina (2007) y citando Medina Fernández de Soto (2009) analiza cómo se consiguen los resultados cuando se optimiza la productividad. Este modelo parte del análisis de la estrategia empresarial, los segmentos objetivos y el mercado, junto con la propuesta de valor, definiendo el nivel de operación y los recursos tangibles e intangibles realmente necesarios.

Adicionalmente, frente a las variables de análisis, las métricas de las buenas prácticas de productividad operacional (BPPO) y las métricas de gestión empresarial son variables cualitativas de orden necesarias en la aplicación de la lógica difusa, identificadas a partir de la importancia entre la productividad y la rentabilidad. Entre ellas están: nivel salarial, productividad laboral, participación del trabajo, productividad del capital, intensidad de capital, razón de utilización del capital, razón de utilización operativa y valor agregado, razón de la utilidad operativa y capital operativo (Quintero Garzón \& Sánchez Fernández, 2018); además de las aplicadas con Lean Manufacturing, entre otras.

Finalmente, y bajo este contexto, la asignación de valores a cada una de las categorías en el proceso de toma de decisiones se puede determinar y evaluar, particularmente en el criterio de decisión (ver Tabla 2). Como lo indica Ruvalcaba \& Vermonden (2015) los valores asignados relacionados a un cierto grado de pertenencia seguirían un continuum, de manera que el criterio seleccionado en la asignación de valores depende del grado de competitividad empresarial (ver Tabla 3).

Tabla 2. Valores asignados a la competitividad

Table 2. Values assigned to competitiveness

\begin{tabular}{cccc}
\hline \multicolumn{4}{c}{ Competitividad } \\
\hline Valor & Insolvencia & Pertenencia & Solvencia \\
\hline 1.1 o menos & $X$ & & \\
\hline 1.2 a 2.9 & & $X$ \\
\hline 2.99 o más & &
\end{tabular}

Tabla 3. Valores asignados a la categoría toma de decisiones Table 3. Values assigned to the decision-making category

\begin{tabular}{cc}
\hline Grado de competitividad & Grado de pertenencia \\
\hline 1.00 & 0 \\
\hline 1.20 & 0.2 \\
\hline 1.80 & 0.4 \\
\hline 2.40 & 0.6 \\
\hline 2.80 & 0.8 \\
\hline 3.00 & 1.0 \\
\hline \multicolumn{2}{c}{ Fuente: elaboración propia. }
\end{tabular}


Para la definición de la unión e intersección de los conjuntos, las funciones pueden generalizarse con la condición que cumplan ciertas restricciones lógicas. Las restricciones están dadas por las funciones: conorma triangular (t-conorma), que es la unión, y norma triangular (t-norma), que se interpreta como la intersección. Los operadores lógicos que cumplen con estas condiciones para ser t-conormas y t-normas, son el operador máximo y la suma algebraica $(O)$ y el operador mínimo y el producto algebraico $(Y)$ respectivamente (Ross, 2004).

De igual forma, para representar el conjunto difuso en análisis, se expresa la función de pertenencia y se mapean los elementos del conjunto con su grado de pertenencia (insolvencia-solvencia). Aunque puede usarse cualquier tipo de función, en la práctica se emplean funciones lineales con una descripción de su vector de ajuste, «bajo-muy bajo» y «alto-muy alto», como se puede ver en la Figura 2.

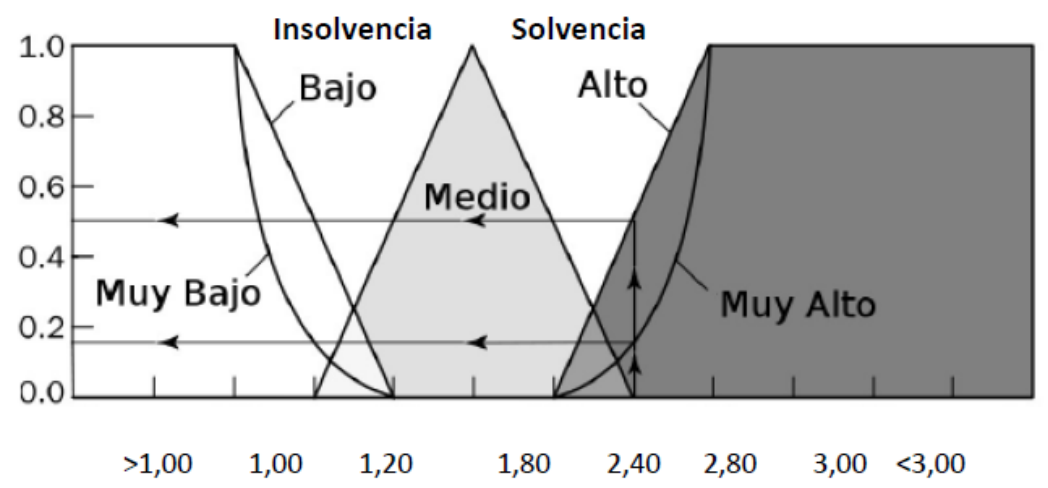

Figura 2. Modificador muy en los conjuntos bajo (insolvencia) y alto (solvencia)

Figure 2. "Very" modifier in Low (insolvency) and High (solvency) sets Fuente: elaboración propia a partir de (Morcillo Loro, 2017).

Para representar los criterios de decisión difusos se utilizan variables lingüísticas, en este caso los conjuntos insolvencia y solvencia y los operadores difusos son verbos y sujetos que, interpretados desde la lógica difusa, son identificados como variables lingüísticas. De esta forma, la variable seleccionada representa cualquier momento temporal de análisis sin importar que sea demasiado complejo para la organización, es decir, lo que no podemos describir en términos cuantitativos. Ya que acudimos a métricas de productividad operacional, al formular las reglas se usan enunciados condicionales de tipo «muy» y "alto», además del término compuesto «muy alto», que compete a la lógica difusa.

El grado de pertenencia es el valor de evaluación y calificación donde cada valoración tiene un porcentaje del valor total y a cada valor se les asigna tantos niveles de la métrica identificada sea posible para la realización del mismo. Para el caso de análisis, se le otorga un valor decreciente: desde $\leq 1,1$ hasta $\geq 2,99$ como el máximo establecido en la métrica. Se pueden establecer niveles mínimos para una evaluación positiva, los cuales compensan las incongruencias generadas por la no realización del objetivo de la métrica, logros imprescindibles desde el enfoque global de la productividad operacional.

Todas las reglas que tienen el mismo consecuente, una vez elegido su operador, definirán el conjunto implicado, debido a que el conjunto se construye para todos los valores que pueda tomar el 
antecedente de la métrica BPPO (Pérez Pueyo, 2016). Del conjunto analizado e implicado se obtienen las métricas con un valor concreto del grado de pertenencia para cada una, con la intersección de los conjuntos (insolvencia e insolvencia) se obtiene el valor del antecedente. Según la literatura analizada, hay dos formas de construir los sistemas de inferencia difusa, una se denomina Mamdani and Assilian (1975), y la otra Sugeno (1985). Estas expresan que las salidas también son conjuntos difusos, y que las salidas son funciones lineales o constantes, respectivamente.

Como último paso en un sistema de inferencia difuso es la 'desfuzzificación'. El cual consiste en obtener un valor numérico de las salidas para los conjuntos difusos a los que pertenecen. Es decir, se obtendrá el valor de la métrica deseada BPPO, y una vez se observen las salidas para los conjuntos, se podrá decir si la decisión a tomar es la adecuada (Fernández et al., 2015). Los métodos más utilizados son:

- El Método del Centroide utiliza el centro de gravedad de la función de pertenencia a partir de las funciones de pertenencia de las salidas (métricas BPPO) y los pesos de cada una de estas. Con este método se obtiene una salida única.

- El Método del Semifallo (Singleton) calcula un promedio de los centroides de las funciones de pertenencia (métricas BPPO) de los conjuntos activados. Luego la ponderación se recurre a los pesos de pertenencia de la salida a los subconjuntos correspondientes.

- El Método de la Media Ponderada se basa en el cálculo del promedio entre las salidas que se obtendrían de los conjuntos difusos multiplicados (ponderados) por el grado de pertenencia al subconjunto.

\section{Contexto de la problemática en las Pymes}

La toma de decisiones constituye un proceso imprescindible para el adecuado y acertado funcionamiento de una organización (Vélez-Salazar \& Duque-Osorio, 2017). En este sentido, muchos estudiosos como Shull et al., (1970); Bourgeois y Eisenhardt (1988); Nutt (1993); Murnighan y Mowen (2002), afirman que el desempeño organizacional optimo requiere una toma de decisiones e implementación eficiente (Rodríguez Cruz \& Pinto Molina, 2010). En la literatura se pueden encontrar numerosos trabajos que analizan la toma de decisiones estratégicas, entre los que destacan los realizados por Mintzberg et al., (1976), Evans y Fischer (1992) y Yung et al., (2006), quienes basan sus trabajos de la toma de decisiones en aspectos solamente del entorno del negocio y en desarrollo de productos.

En este contexto, las buenas prácticas de productividad operacional (BPPO) se definen como un conjunto de metodologías y herramientas que ayudan a las empresas a mejorar e incrementar su productividad a través de su incorporación en el hacer diario y su apropiación organizacional, aspectos que todavía esperan a ser incluidos para la toma de decisiones (Nasir et al., 2016). Bajo esta perspectiva, existen en el mundo muchos tipos de buenas prácticas productivas, como las que se proponen en la manufactura esbelta ( $5 \mathrm{~s}$, jidoka, kanban, VSM, kaizen, andón, entre otras). La toma de decisiones empresariales y su relación frente a las BPPO no ha sido considerada. En razón a lo anterior, las aproximaciones teóricas y metodológicas existentes muestran la falta de métodos formales que ayuden a comprender el fenómeno, principalmente por la falta de la integración de diferentes tipos de variables, su análisis matemático integral y la particularización por sector económico interesado (Grundey \& Heeks, 1998; Tschang, 2003). 
Esta brecha de conocimiento en las propuestas debe ser cerrada para avanzar de forma segura en la construcción de sectores empresariales competitivos. Se espera aportar con la contextualización de la lógica difusa relacionada a las BPPO que cumpla con las características básicas ya planteadas en la literatura y logre satisfacer las limitaciones de los métodos e instrumentos existentes. Asimismo, contribuyen a cerrar la brecha y permiten a los empresarios conocer el estado relacional entre la empresa, la productividad y el mercado con el fin de alcanzar estados de futuro competitivos.

Las Pymes en la era del conocimiento y la información enfrentan retos y desafíos para la acertada toma de decisiones, que, por su estructura orgánica, evidencian debilidad y falta de métodos que proporcionen mejores resultados en el desempeño de la organización. Consideraciones como la calidad, el costo y cumplimiento de factores influyentes en las decisiones se han convertido en atributos no suficientes para competir con éxito en los mercados actuales (Cabeza de Vergara \& Muñoz, 2010).

Precisamente, esta eficiente y efectiva implementación recae en manos de aquellos individuos que tienen la responsabilidad de tomar decisiones, es decir, los directivos de las organizaciones (Rodríguez Cruz, 2014). Por lo tanto, es necesario aportar en líneas de investigación, orientaciones metodológicas que apoyen la toma de decisiones empresariales. El nuevo gerente debe ser adaptativo, con altísima capacidad de respuesta y en condiciones intelectuales para pensar más allá de lo inimaginable (Ludovic, 1995).

De acuerdo con Buitrago Quintero (2007), en el futuro de las organizaciones en sus métodos de análisis deben permitir, elegir entre diversas opciones estratégicas. La toma de decisiones debe hacerse en condiciones de concertación y consenso entre los directivos, teniendo en cuenta la cultura al interior de la organización y el entorno que lo afecta. Además de considerar factores que influyen en la competitividad como:

menor tiempo de entrega de nuevos productos, más ciclos de inventarios, tiempo de entrega por fabricación más corto, calidad más alta, mayor flexibilidad en la fabricación, mejor servicio a clientes, menores niveles de desperdicios, mayor rendimiento de los activos (González Santoyo et al., 2012).

Las pequeñas y medianas empresas deben adoptar posiciones estratégicas mediante la toma de decisiones «oportunas que soporten plenamente los objetivos de la empresa, permitiendo reaccionar de forma rápida y flexible ante los acontecimientos externos, interviniendo en toda la organización» (Infante Quiroz, 2010) en temas como la transferencia de conocimiento y tecnología relacionados con la efectividad de las BPPO.

Se hace énfasis en que la pequeña y mediana empresa requiere de métodos en la toma de decisiones empresariales que le permitan hacer presencia en forma competitiva en los espacios regionales de generación de riqueza, cosa que motiva a realizar este artículo, dado que su validación con empresas del sector de manufactura reflejaría el hecho de un posible acierto en decisiones estratégicas empresariales, y los resultados del mismo podrían ser insumo para innovar y propiciar el salto de pequeña y mediana empresa, a empresas dinámicas y de alto impacto. Por esto, es fundamental que la organización genere constantemente procesos de vigilancia del entorno, que lo orienten en su 
quehacer y le permitan mantener su actitud proactiva y construir el futuro, evolucionando con creatividad el mañana donde sus actuaciones logren el futuro deseado (Buitrago Quintero, 2007).

Frente al enfoque, González (2013) plantea que, basado en procesos, es un conjunto de actividades mutuamente relacionadas o que interactúan, cuyos elementos de entrada se ven transformados en resultados, cosa que posibilita a la compañía efectuar un análisis de perfeccionamiento de las funciones y actividades de sus colaboradores para lograr mejoramiento en su competitividad. De esta forma, las organizaciones, para operar de manera eficaz, identifican y gestionan numerosos procesos interrelacionados. Se espera, por medio de la práctica experimental empresarial, contribuir desde esta mirada a las Pymes del sector de manufactura en la toma de decisiones apropiadas basadas en la productividad, involucrando métricas específicas que relacionen las BPPO en la ciudad de Medellín. Desde esta perspectiva, es válido mencionar que las Pymes manufactureras seleccionadas para el estudio han trabajado diferentes programas de mejoramiento productivo con organizaciones como la Cámara de Comercio, el Centro de Ciencia y Tecnología de Antioquia (CTA) o Colombia Productiva (antes PTP), convirtiéndolas en apropiadas para el análisis propuesto, además han presentado mejoras en sus áreas de producción al aplicar las BPPO como mecanismo de mejora continua.

Frente a la conceptualización de la toma de decisiones, Canós et al., (2000) plantean que «una decisión es la elección de la alternativa más adecuada de entre varias posibilidades con el fin de alcanzar un estado deseado, lo anterior considerando la limitación de recursos». Canós et al., (2000) considera la toma de decisiones como un proceso en el que se integran la inteligencia, el diseño, la selección, la implementación y la revisión (De Miguel, 1993; Moody, 1991; Huber, 1984; Simon, 1977). En este sentido, las personas encargadas de tomar una decisión son los decisores (Davis, 2000), que serán los directivos u otros empleados de la empresa en función del tipo de decisión.

Adicionalmente, frente a las características de la toma de decisiones, se debe aclarar inicialmente que la información es el principio y el fin del ciclo Información-Decisión-Acción, (Claver Cortés et al., 2000). Con información adecuada se puede tomar una decisión que impulsa a la implementación de una acción. Esta acción genera nueva información con la que se retroalimenta el proceso y se vuelve a iniciar la necesidad de tomar nuevas decisiones. Es de resaltar que todo este ciclo de toma de decisiones se lleva a cabo bajo un contexto de ambientes dinámicos.

\section{METODOLOGÍA}

Con respecto a la metodología, se hace desde un enfoque descriptivo utilizando como métrica fundamental el índice de productividad total de factores (IPTF), que agrupa las variables fundamentales que requiere una organización para medir su productividad real, entre ellos volumen de ventas, precio de venta unitario, costo de mano de obra, número de empleados, costo de materia prima y depreciación.

Se examinan diferentes métricas para la gestión empresarial, conceptos de lógica difusa y buenas prácticas operacionales (BPPO) planteadas por los investigadores de este campo con el fin de explicar y describir los elementos que apoyan la lógica difusa para la toma de decisiones desde la potencialidad efectiva de su contribución a la productividad empresarial. Además, se analizan los resultados de la intervención a 6 Pymes ya anunciadas, cuyo estudio se enfoca en las dinámicas 
internas, sus componentes, su problemática y sus necesidades, entre otras prácticas, como logros permanentes y rutinarios de las organizaciones.

Con respecto a la obtención del IPTF, se utilizó la construcción de los índices de insolvencia y solvencia como ejes centrales del análisis, donde una empresa con nivel competitivo es considerada solvente cuando su valor es igual o superior a 2.99, por lo que una empresa cuyo valor se esté alejando al valor 2.99 va perdiendo pertenencia al conjunto de solvencia, y, en cambio, ganando pertenencia al conjunto de insolvencia. Lo anterior, complementado con índices como el ciclo financiero, el margen EBITDA y la palanca de crecimiento como outputs que, asociados a los inputs, indicadores de productividad laboral (BPPO-OEE), permiten a las organizaciones tomar decisiones de acuerdo al IPTF semestral o anual obtenido. Para el caso citado, se realiza el ejercicio para el período 2016-2017 en 6 Pymes del sector industrial. Es de considerar que, en virtud de las restricciones de información del nombre de las empresas, estas pertenecen a los sectores metalmecánico, autopartes y comunicación gráfica.

Una vez realizada la intervención en las empresas, se evalúan los resultados y se establecen las relaciones conceptuales que fundamentan la discusión y conclusiones bajo el contexto de la toma de decisiones adecuadas y su aplicación con la lógica difusa a partir de la síntesis desarrollada. Así, se evidencia la utilidad de la lógica difusa y las BPPO como una alternativa para la toma de decisiones empresariales.

Para el desarrollo de la investigación se plantean diferentes fases. En la primera, se define el problema de decisión y los criterios para la toma de decisión. Luego se establece el decisor y el enunciado verbal seguido del predicado y en la Figura 3 muestra la secuencia a seguir en la decisión final.

Problema de decisión identificado: ¿Cuál alternativa a seleccionar que contribuya, en mayor medida, a elevar la competitividad de la empresa dentro del sector de manufactura en Medellín-Colombia?

Criterios para la toma de decisión (0.1) para determinar la membresía de un grupo:

1. Se mejora el estado financiero.

2. Se incrementa la participación de mercado.

3. Se incrementan las ventas.

4. Se incrementa la calidad de los servicios.

5. Se incrementan las ventas.

6. Se mantienen buenas relaciones de pago con los proveedores. (Brunet Cortina \& Robaina, 2010).

\section{Decisores: el gerente de cada empresa o comité decisor, constituido para su total aprobación.}

\section{Enunciado verbal}

La empresa se hace más competitiva si incrementa la satisfacción del cliente, la de los proveedores, y si se aleja de la insolvencia económica.

- Se incrementa la satisfacción de expectativas de clientes, si cumple los plazos de entrega con efectividad en el servicio.

- Incrementa la satisfacción de proveedores, si incrementa la cuota de mercado y mantiene buenas relaciones de pago. 
- Reduce la insolvencia económica si mejora su estado financiero o aumenta en gran medida las ventas con margen de rentabilidad positivo.

Predicado, de acuerdo al desarrollo de los criterios decisores dados por Brunet Cortina y Robaina, (2010), se pueden agrupar bajo los siguientes criterios:

C(x) La empresa eleva su competitividad.

$\mathrm{EC}(\mathrm{x})$ Se incrementa la satisfacción de consumidores.

$\mathrm{EP}(\mathrm{X})$ Se incrementa la satisfacción de proveedores.

$\mathrm{SE}(\mathrm{x})$ Se incrementa la solidez económica.

$\mathrm{Pe}(\mathrm{x})$ Se mejora el indicador de cumplimiento de los plazos de ejecución.

$\mathrm{Cs}(\mathrm{x})$ Se incrementa la calidad de los servicios.

$\mathrm{Cm}(\mathrm{x})$ Se incrementa la cuota de mercado.

$\mathrm{Rp}(\mathrm{x})$ Se mantienen buenas relaciones de pago.

$\mathrm{Ef}(\mathrm{x})$ Mejoran los estados financieros.

$\mathrm{V}(\mathrm{x})$ Incrementan las ventas.

La decisión será tomada según el árbol que se muestra en la Figura 3. Toma de decisiones, donde a través de una operación conjuntiva, se pueden mejorar los niveles de competitividad de la organización con decisiones más acertadas.

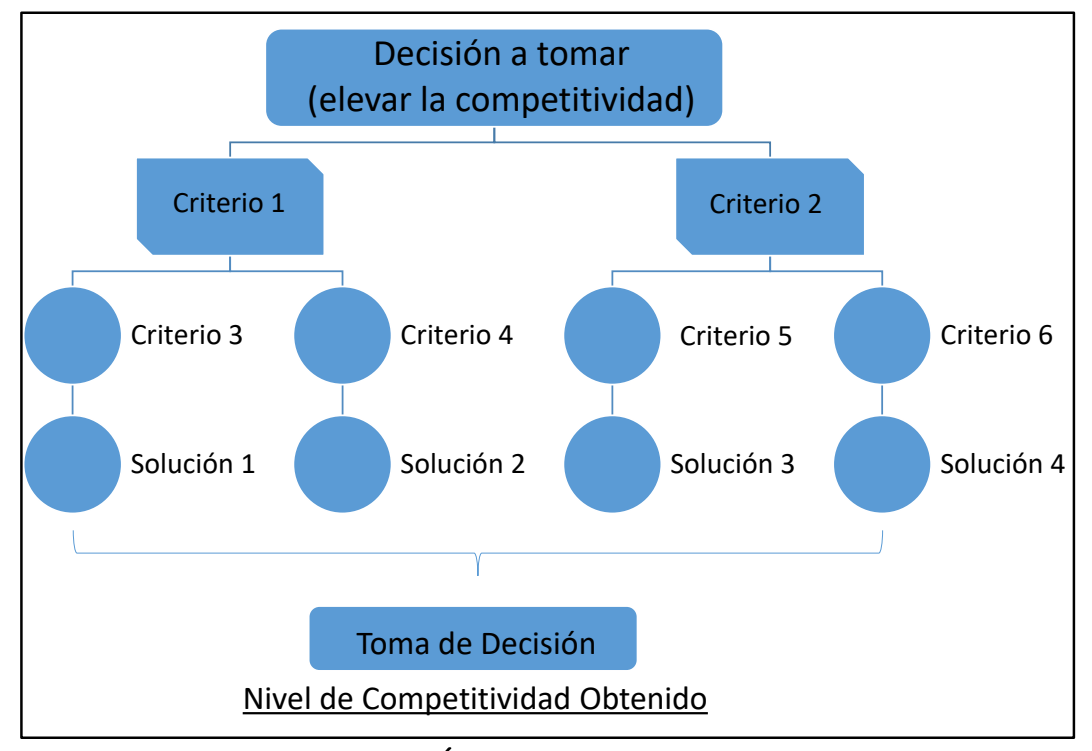

Figura 3. Árbol Toma de decisiones

Figure 3. Decision-making tree

Fuente: elaboración propia.

\section{RESULTADOS}

Como se ha indicado en la Tabla 2 y Tabla 3, se integran los valores que, por su objetivo y contenido, recuperan la lógica difusa original de Zadeh y cuya aplicación está relacionada con la toma de decisiones empresariales adecuadas. Así, se cumple el objetivo del presente documento de exponer la aplicación de la lógica difusa como herramienta para medir la certeza de la toma de decisiones 
conforme a la efectividad de la aplicación al problema que enfrenta el decisor, por lo cual es posible referenciar de manera general la utilización de la lógica difusa y los resultados en las conclusiones ofrecidos por el autor.

Así mismo, se presenta una propuesta concreta para la incorporación de la lógica difusa en el proceso final de la toma de decisión basado en la productividad, otorgando valores específicos a los rasgos evaluados.

A continuación, se presentan los resultados de los análisis IPTF realizados a las 6 Pymes del sector industrial. Como consideración inicial, los datos considerados por el Índice de Productividad Total de Factores como estándares de referencia para las organizaciones competitivas se relacionan en la Tabla 4 (OECD, 2001).

Tabla 4. Datos IPTF

Table 4. Information regarding TFP

Índice de Productividad Total de Factores

$\begin{array}{r}\hline \text { iptf } \geq 3 \text {, Nivel World Class. Situación muy competitivo } \\ \hline \text { iptf } \geq 2<3 \text {, Nivel muy bueno. Situación competitivo } \\ \hline \text { iptf } \geq 1<2 \text {, Nivel Bueno. Situación Aceptable } \\ \hline \text { iptf } \leq 0.5<1 \text {, Nivel Regular. Situación aceptable solo con mejora } \\ \hline \text { Fuente: elaboración propia a partir de OECD (2001). }\end{array}$

En la Tabla 4 se presentan los niveles de pertenencia que enmarcan los grados de competitividad empresarial, siendo el IPTF $\leq 0.5$, el grado en que requiere la PYME mayor intervención y el IPTF $\geq 3$ donde la PYME evidencia un estado de competitividad alto y llega a nivel de clase mundial.

Para establecer un hilo conductor de análisis, se definieron indicadores entre los que interactúan la productividad laboral (BPPO) y los resultados de desempeño financieros, con el fin de relacionar directamente la importancia de la toma de decisiones con el IPTF y los valores obtenidos por las organizaciones objeto de estudio durante su comportamiento anual entre el 2016 y 2017. Estos indicadores se identifican en la Tabla 5.

Tabla 5. Indicadores para el IPTF

Table 5. TFP indicators

\begin{tabular}{ccccc}
\hline \multirow{2}{*}{ Empresa } & Ciclo financiero (días) & Margen Ebitda & Palanca crecimiento & oee \\
\hline 1 & 151 & $0.88 \%$ & 0.0772 & $49.56 \%$ \\
\hline 2 & 196 & $3.92 \%$ & 0.1100 & $57.00 \%$ \\
\hline 3 & 220 & $14.47 \%$ & 0.5707 & $71.26 \%$ \\
\hline 4 & 195 & $4.96 \%$ & 0.1123 & $63.78 \%$ \\
\hline 5 & 15 & $22.11 \%$ & 8.1200 & $70.98 \%$ \\
\hline 6 & 139 & $3.75 \%$ & 0.0953 & $80.06 \%$ \\
\hline \multicolumn{5}{c}{ Fuente: elaboración propia. }
\end{tabular}


Una vez consolidados los resultados de los indicadores de la Tabla 5 y ejecutadas las actividades propias de mejoras de la organización por sus áreas de producción, el área financiera complementó los datos con el indicador de Insolvencia y Solvencia para tabular correctamente el IPTF final y poder realizar la comparación con respecto a los valores de referencia de la Tabla 4. A continuación, se presentan en la Tabla 6 los valores finales por organización del Índice de Productividad Total de Factores.

Tabla 6. Índice obtenido IPTF

Table 6. Estimated TFP

\begin{tabular}{cccc}
\hline Empresa & Insolvencia & Solvencia & iptf \\
\hline 1 & 2.383 & 1.38 & 2.525 \\
\hline 2 & 2.259 & 2.95 & 2.130 \\
\hline 3 & 2.589 & 1.92 & 2.865 \\
\hline 4 & 2.188 & 1.14 & 1.405 \\
\hline 5 & 5.146 & 1.94 & 1.760 \\
\hline 6 & 2.275 & 2.07 & 1.035 \\
\hline \multicolumn{4}{c}{ Fuente: elaboración propia. }
\end{tabular}

Como se puede observar en la Figura 4, se relacionan las gráficas del indicador IPTF obtenido después de la intervención en las empresas manufactureras y como resultado de las diferentes acciones empresariales y que son factores de pertenencia en los criterios de la aplicación de la lógica difusa para la toma de decisiones. Estos indicadores permitieron a las empresas y sus decisores tomar acciones para el mejoramiento de la productividad. Convención: semestre 1 (S1) y semestre 2 (S2).

Empresa 1: IPTF: 2.72 (S1) 2.33 (S2)

Promedio obtenido en el año: IPTF 2.525
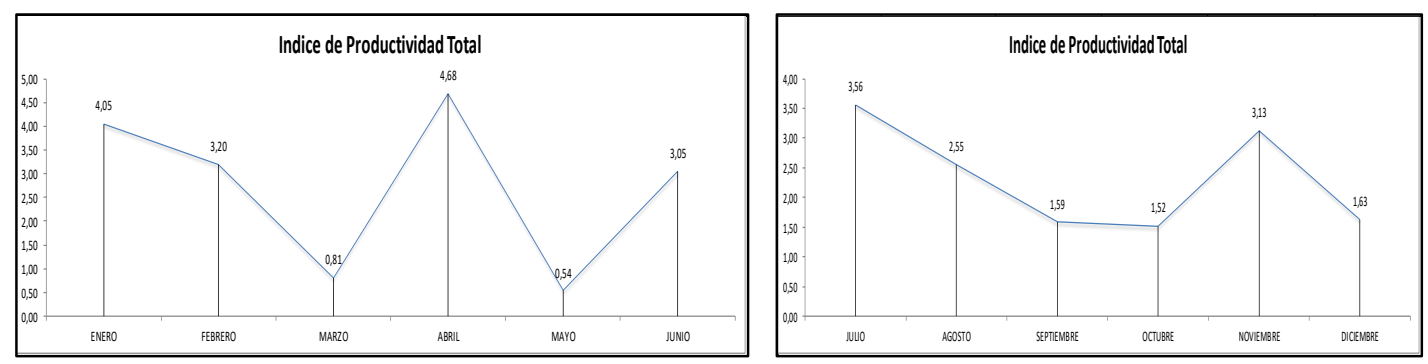

Empresa 2: IPTF: 0.88 (S1) 3.38 (S2)

Promedio obtenido en el año: IPTF 2.13 

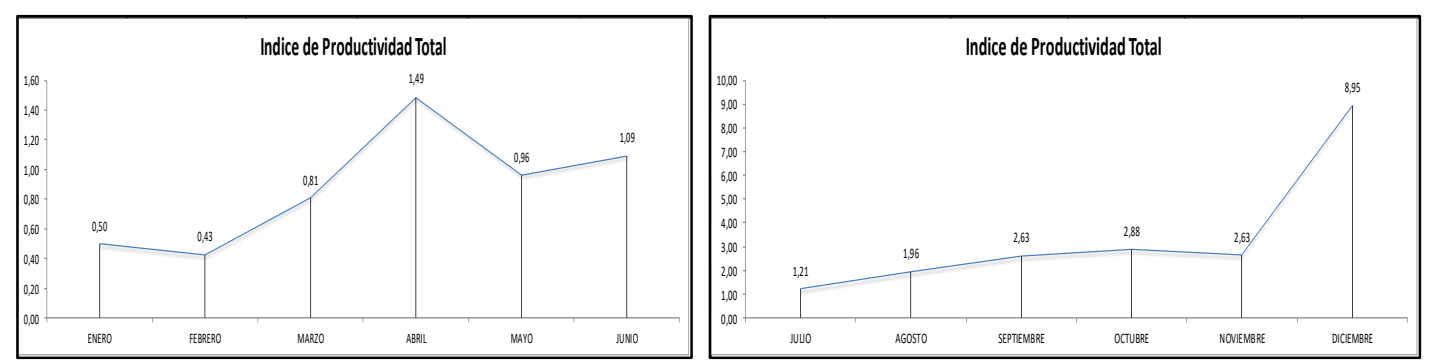

Empresa 3: IPTF: 1.82 (S1) 3.91 (S2)

Promedio obtenido en el año: IPTF 2.865
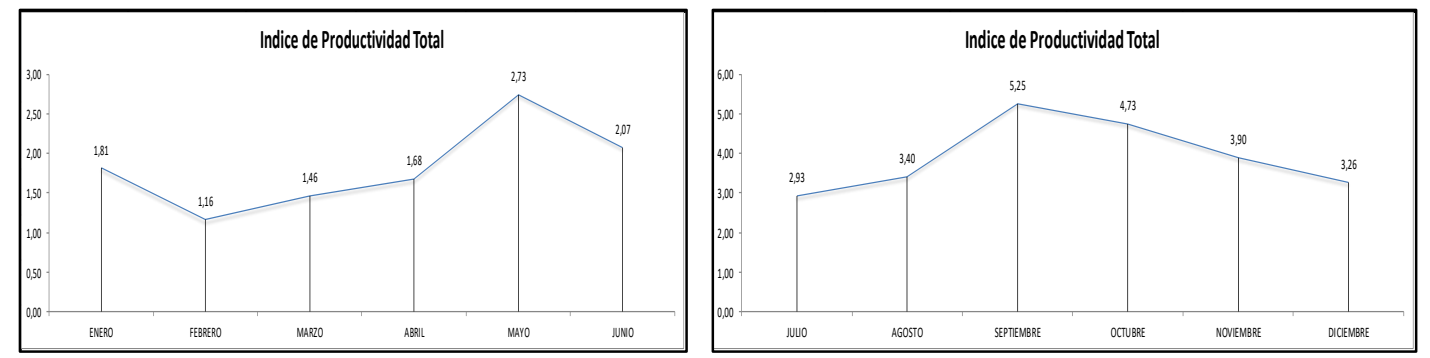

Empresa 4: IPTF: 1.27 (S1) 1.54 (S2)

Promedio obtenido en el año: IPTF 1.405
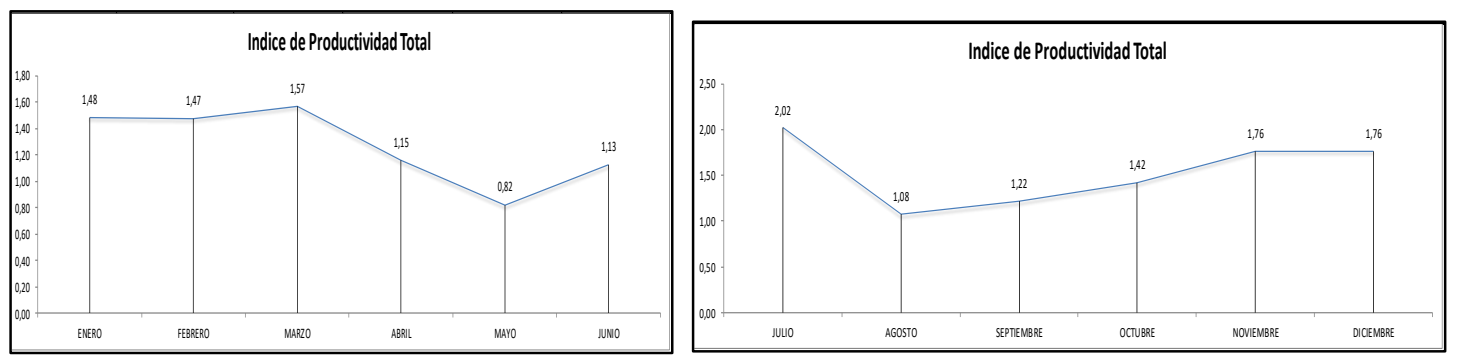

Empresa 5: IPTF: 1.68 (S1) 1.84 (S2)

Promedio obtenido en el año: IPTF 1.76
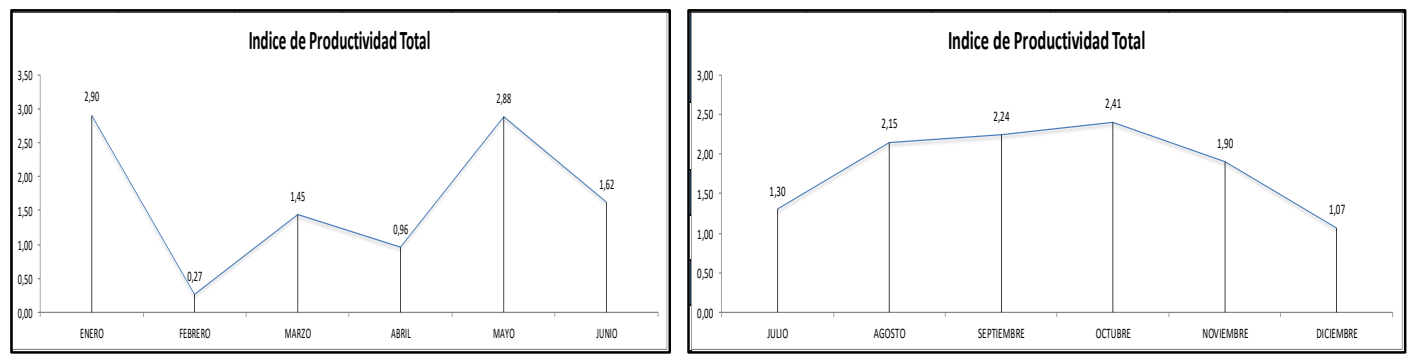

Empresa 6: IPTF: 0.91 (S1) 1.16 (S2) 

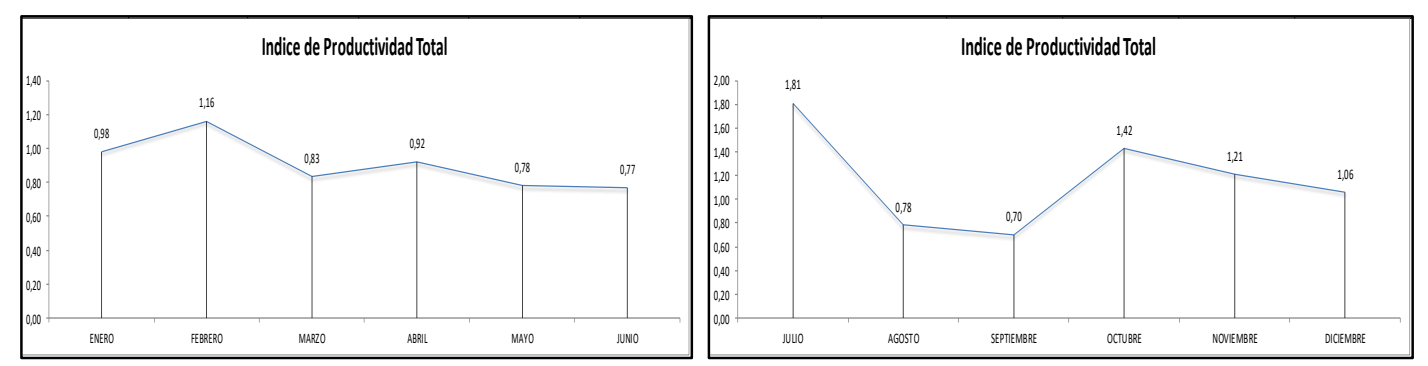

Figura 4. Niveles IPTF obtenido de las empresas.

Figure 4. Estimated TFP levels for each company Fuente: elaboración propia.

A la vista de los desarrollos expuestos y con la cautela que exige la obtención de resultados fundamentados en lo sucedido en estudio, se puede destacar que, en el sector manufacturero, a la luz de la intervención durante el periodo 2016-2017 en relación con los indicadores de productividad (Figura 4) obtenidos de las empresas, pueden observarse con cierta nitidez dos subconjuntos de interpretación. Están aquellos que obtuvieron unos resultados ajustados a la media IPTF $\geq 1<2$ y que aglutina el $50 \%$ de las empresas, un grupo con niveles de productividad inferiores a la media, IPTF $\geq$ $1<2$ e integrado por niveles cercano, medio y bajo a la media de las empresas; y por otra parte un grupo en la media IPTF $\geq 2<3$ con niveles cercano, medio y bajo del valor superior IPTF $\geq 3$. Lo que indica que el $50 \%$ de las organizaciones objeto de estudio requieren de un plan de mejora permanente, mientras que el otro $50 \%$ requiere de mantener los planes de mejora para sostener su productividad, además de aclarar que para ambos casos no caer en estados de insolvencia en escenarios futuros es imprescindible.

Igualmente, las variables que mayor impacto negativo provocan sobre el IPTF serían: el ciclo financiero y el margen EBITDA medido por periodos mínimos semestrales o anuales, que son parte de los inputs, donde el índice de la productividad total de factores se ve afectada negativamente por el radio de insolvencia-solvencia, que son outputs, para lo cual las variables que más favorecen el IPTF la proporcionan la calidad, disponibilidad y rendimiento de los recursos endógenos de la organización.

Bajo este contexto, la valoración de los datos Tabla 6, es decir, inputs de los indicadores de Insolvencia y Solvencia, afectan el IPTF como resultante de la combinación de las acciones internas. Esta situación permitió a las Pymes tomar decisiones referidas a la implementación de esquemas o sistemas que mejoren la BPPO y eleven la competitividad organizacional.

Por último, los resultados del estudio de las 6 Pymes durante el periodo 2016-2017 indican que la toma de decisiones de las organizaciones fue significativa para el mejoramiento productivo global en la medida que estas organizaciones controlaron el IPTF y mantuvieron como objetivo la eficiencia global óptima. Esto permitió mantener las condiciones más cercanas a los principios difusos confirmando la efectividad de la lógica difusa para el proceso de toma de decisiones empresariales. 


\section{DISCUSIÓN}

El trabajo desarrollado plantea como objetivo examinar la toma de decisiones basadas en la productividad en Pymes manufactureras a través de la utilización de lógica difusa basada en métricas de productividad operacional (BPPO), y, por otro lado, el aprovechamiento de la lógica difusa en el proceso de toma de decisiones en el escenario organizacional o empresarial. Además, como es conocido en diversos textos referenciados a Zadeh (1965) se plantean «las limitaciones de la asignación binaria (0.1) para determinar la membresía de un grupo. Para este autor, esta pertenencia a conjuntos no es absoluta», sino permanente y continua, va desde cero hasta uno. Desde el caso de las 6 Pymes, los valores de la Tabla 2 y Tabla 3 mostraron que las operaciones que están contenidas en el desarrollo de la intervención podrían ser también explicadas desde la lógica difusa, a saber: unión, complementación e intersección (Figura 1). En razón a esto, bajo los principios difusos, es importante expresar que la toma de decisiones empresariales efectivas, por sí sola, genera incertidumbre.

Las aplicaciones expuestas en los documentos de la Tabla 4, desde Bellman y Zadeh (1970) hasta Merigó y Peris-Ortiz (2014), muestran la asignación de valores concretos en lógica difusa para el proceso de toma de decisiones, con la interdependencia de los valores a cada variable deseada para la toma de decisión empresarial. Esto permite tomar una decisión basada exclusivamente en resultados cuantificables dados por las métricas de productividad operacional. La eliminación de vaguedad, imprecisión y ambigüedad en el proceso de selección de toma de decisiones es fundamental para estos autores, quienes ven útil y factible el modelo difuso, incluso para situarse al ritmo de las exigencias contemporáneas de eficiencia operacional.

La utilización de métricas BPPO son consistentes para obtener información como resultados concretos, pero es fundamental la modelación matemática que transforme esas métricas en valores para procesar matemáticamente la decisión, ya sea dentro de la empresa o a través de un centro de evaluación externo (Adler, 1987; Thornton \& Gibbons, 2009).

Canós et al., (2011) y De Korvin y Kleyle (2000) utilizan la lógica difusa para detectar la productividad del personal en la empresa: lo más cercano a la eficiencia operativa. Otros autores como Jereb et al., (2005) y Díaz Mora et al., (2009), utilizan el modelo de conjuntos difusos como un proceso analítico jerarquizado. Por su parte, Saaty et al., (2007), Lin (2010) y Saaty y Vargas (2012), lo usan para la selección de empresas contratistas y, en muchos casos, para la selección de personal (Özdaban \& Özkan, 2011). Sin embargo, las implicaciones prácticas para todos estos autores han demostrado la utilidad de la aplicación de la lógica difusa mientras los procesos sean analíticos y jerarquizados, temas aún por profundizar en su efectividad y alcance.

Aunque las publicaciones que dan cuenta del uso de la lógica difusa de Zadeh (1965) son numerosas, en lo referido al contexto empresarial, se reducen significativamente. Esto evidencia que aún está en desarrollo para nuevas propuestas en ámbitos empresariales, más en contextos de globalización y mercados con entornos de incertidumbre, y que la lógica difusa es vista ya como una herramienta factible y útil, no solo para el proceso referido, sino para otros que se presentan en el escenario empresarial. 
Para los diferentes sectores económicos como el industrial, comercial, construcción, minero energético, financiero, entre otros, la lógica difusa abre una serie de posibilidades, siendo las variables seleccionadas las que podrían permitir una calificación de permanencia aprovechando los efectos del entorno como factor determinante. Es de considerar que la definición de criterios a estudiar puede variar en mayor o menor medida a través de la incorporación de los indicadores globales de la organización. Esto señala que la aplicación de la lógica difusa podría contribuir a una mayor objetividad en la toma de decisiones.

Finalmente, la lógica difusa ofrece una flexibilidad y rigidez suficientes, como lo expresa Güngör et al., (2009), para ajustarse a diferentes modelos, así como a procesos más amplios. Esto permite orientar y dar pauta a su uso innovador bajo diversas metodologías para la toma de decisiones.

\section{CONCLUSIONES}

Para el autor la realización de este trabajo se facilitó gracias a la información obtenida en el diagnóstico inicial a las empresas y el uso de indicadores asociados al desempeño de la organización, lo que permitió establecer los criterios decisores y el enunciado verbal para la toma de decisión, lo que permitan elevar la competitividad y sostenibilidad de la organización en el mercado. Esto gracias a la vinculación de las BPPO en la toma de decisiones organizacionales.

Con el análisis realizado, y contrastando los resultados con el objetivo propuesto se puede afirmar que la lógica difusa aplicada a las variables de productividad, en este caso las BPPO y el modelo IPTF permiten brindar certidumbre en la toma de decisiones empresariales, lo que significa para los tomadores de decisiones una mayor efectividad en la decisión final.

De igual forma los resultados obtenidos del estudio deben ser considerados con cautela a la luz de las limitaciones que son propias del desarrollo metodológico, por una parte, la generalización de las métricas utilizadas corresponden a un universo de 6 PYMES manufactureras de la ciudad de Medellín y en segunda instancia la utilización de la lógica difusa y las dimensiones definidas permite que los resultados en su valoración cuantitativa reflejan la reducción de incertidumbre como pretende el objetivo propuesto.

Bajo este contexto, en el análisis del árbol de decisión bajo criterios difusos, los indicadores de desempeño toman un papel fundamental en la toma de decisión final, lo que demuestra que la toma de decisión basada en resultados cuantificables BPPO es fundamental para generar certidumbre. Así la asignación grupal de cero (insolvencia) hasta uno (solvencia) en la toma de decisiones, permite la utilización de técnicas para obtener información, como registros manuales o software bien estructurados, premisas útiles para transformar esos datos en información que facilita su valoración para la toma de decisiones.

Finalmente, el uso de lógica difusa abre un mundo de posibilidades para estudios de nuevas herramientas frente a las metodologías para la toma de decisiones empresariales, respecto al adecuado manejo administrativo que permita a las organizaciones sostenibilidad, perdurabilidad y competitividad en el tiempo. Una línea futura de trabajo en la toma de decisiones empresariales podría referirse a la realización de un análisis de sensibilidad sobre los conjuntos de pertenencia para las BPPO. 


\section{REFERENCIAS}

Adler, S. (1987). Toward the more efficient use of assessment center technology in personnel selection. Journal of Business and Psychology, v. 2, n. 1, 74-93. https://doi.org/10.1007/BF01061526

Altman, E. I. (1968). Financial Ratios, Discriminant Analysis and the Prediction of Corporate Bankruptcy. Journal of Finance, v. 23, n. 4, 589-609. https://doi.org/10.2307/2978933

Arango-Serna, M. D.; Serna Durán, C. A.; Pérez-Ortega, G. (2012). La Gestión de Indicadores Empresariales con Lógica Difusa para la Toma de Decisiones. Lámpsakos, v. 1, n. 8, 47-53. https://doi.org/10.21501/21454086.678

Belalcázar, G. R.; Trujillo, O. A. (2016). ¿Es el modelo Z-Score de Altman un buen predictor de la situación financiera de las Pymes en Colombia? (tesis de maestría). Universidad EAFIT, Medellín, Colombia.

Bellman, R.; Zadeh, L. (1970). Decision-Making in a Fuzzy Environment. Management Science, v. 17, n. 4, B141-B164. https://doi.org/10.1287/mnsc.17.4.B141

Bobillo, F.; Delgado, M.; Gómez-Romero, J.; López, E. (2009). A semantic fuzzy expert system for a fuzzy balanced scorecard. Expert Systems with Applications, v. 36, n. 1, 423-433. https://doi.org/10.1016/i.eswa.2007.09.020

Bourgeois, L. G.; Eisenhardt, K. M. (1988). Strategic Decision Processes in High Velocity Environments: Four Cases in the Microcomputer Industry. Management Science, v. 34, n. 7, 816-835. https://doi.org/10.1287/mnsc.34.7.816

Brunet Cortina, I.; Robaina, D. A. (2010). Lógica difusa aplicada a la toma de decisiones. Ingeniería Industrial, v. 31, n.1, 1-5. URL

Brunetto, Y.; Farr Wharton, R. (2007). The moderating role of trust in SME owner/managers' decision making about collaboration. Journal of Small Business Management, v. 45, n. 3, 362-387. http://dx.doi.org/10.1111/j.1540-627X.2007.00218.x

Buitrago Quintero, C. J. (2007). La incertidumbre de futuro y la estrategia empresarial. AD-Minister, n. 11, 1-35. URL

Cabeza de Vergara, L.; Muñoz Santiago, A. (2010). Análisis del proceso de toma de decisiones, visión desde la PYME y la gran empresa de Barranquilla. Cuadernos Latinoamericanos De Administración, v. 6, n. 10, 9-39. https://doi.org/10.18270/cuaderlam.v6i10.1062

Canós, L.; Casasú, T.; Crespo, E.; Lara, T.; Pérez, J. C. (2011). Selección de Personal Basada en Métodos Difusos. Revista de Matemática: Teoría y Aplicaciones, v. 18, n. 1, 177-192. https://doi.org/10.15517/RMTA.V18I1.2122 
Canós Darós, L.; Pons Morera, C.; Valero Herrero, M.; Maheut, J. P. D. (2000). Toma de decisiones en la empresa: proceso y clasificación. $\underline{U R L}$

Cassou, S. P.; Xavier de Oliveira, E. (2011). Barriers to technological adoption in Spain and Portugal. Portuguese Economic Journal, v. 10, n. 3, 189-209. https://doi.org/10.1007/s10258-010-0069-1

Castiblanco Ruiz, F. A. (2013). La incertidumbre y la subjetividad en la toma de decisiones: una revisión desde la lógica difusa. Lúmina, v. 14, 116-141. https://doi.org/10.30554/lumina.14.1086.2013

Chen, G. M. (2010). The Impact of Intercultural Sensitivity on Ethnocentrism and Intercultural Communication Apprehension. Intercultural Communication Studies, v. 19, n. 1, 1-9. URL

Claver Cortés, E.; Llopis Taverner, J.; Lloret Llinares, M.; (2000). Manual de administración de empresas. Civitas.

Davis, D. (2000). Investigación en administración para la toma de decisiones. Thomson.

De Miguel Fernández, E. (1993). Introducción a la gestión (management) I. Universidad Politécnica de Valencia.

De Korvin, A.; Kleyle, R. (2000). A fuzzy set approach to resource procurement for multi-phase projects subject to flexible budgetary constraints. Journal of Intelligent and Fuzzy Systems, v. 9, n. 3-4, 225-233.

Díaz Duarte, D. (2005). Toma de decisiones: el imperativo diario de la vida en la organización moderna. ACIMED, v. 13, n. 3, 8-23. URL

Díaz Mora, R.; Piña, J.; Ríos, D.; Serafín, M. (2009). Uso de AHP y conjuntos difusos para mejorar la toma de decisiones. Caso: Selección de Empresas Contratistas de Construcción en la Administración Pública Venezolana. En San Cristobal: Seventh LACCEI Latin American and Caribbean Conference for Engineering and Technology.

Evans, B. K.; Fisher, D. G. A. (1992). A Hierarchical Model of Participatory Decision-Making, Job Autonomy, and Perceived Control. Human Relations, v. 45, n. 11, 1169-1189. https://doi.org/10.1177/001872679204501103

Fernández, D.; Ordiales, M.; Sancho, J.; Verdeja, L. F. (2015). Posibilidades de la lógica difusa en operaciones y procesos de la metalurgia primaria. En 1a Expo Convención Mundial en Minería, Energía y Metalurgia (Spain Minergy 2015), Julio 17-19, 2015, Gijón, Asturias.

García-Alegre, M. C. (1991) Inteligencia Artificial en el control de procesos, controladores borrosos. Mundo Electrónico, n. 214, 42-49 
Godlee, F.; Pakenham-Walsh, N.; Ncayiyana, D.; Cohen, B.; Packer, B. (2004). Can we achieve health information for all by 2015? The Lancet, v. 364, n. 9430, 295-300. https://doi.org/10.1016/S0140-6736(04)16681-6

González, M. C. (2007). Lógica Difusa: Una introducción práctica. Técnicas de Softcomputing, 1-29. $\underline{U R L}$

González Santoyo, F.; Flores Romero, B.; Chagolla Farías, M. (2012). Herramientas de productividad como base para el diseño de estrategias de mejoras empresariales. Ciencias Empresariales, n. 5, 16. $\underline{U R L}$

González, H. (2013). Enfoque basado en procesos como principio de gestión. URL

Grundey, M.; Heeks, R. (1998). Romainia's hardware and software industry: building IT policy and capabilities in a transition economy. Institute for Development Policy and Management, University of Manchester. Oxford, U.K.

Güngör, Z.; Serhadlıoğlu, G.; Kesen, S. E. (2009). A fuzzy AHP approach to personnel selection problem. Applied Soft Computing, v. 9, n. 2, 641-646.

https://doi.org/10.1016/i.asoc.2008.09.003

Huber, G. P. (1984). Toma de decisiones en la gerencia. Trillas.

Infante Quiroz, M. del C. (2010). Modelos de innovación para la mejor organización del departamento de recursos humanos en el área administrativa en empresas pymes. $\underline{U R L}$

Jereb, E.; Rajkovic, U.; Rajkovic, V. (2005). A Hierarchical Multi-Attribute System Approach to Personnel Selection. International Journal of Selection and Assessment, v. 13, n. 3, 198-205. https://doi.org/10.1111/j.1468-2389.2005.00315.x

Khan, K. U.; Atlas, F.; Xuehe, Z.; Khan, F.; Khan, S. (2019). The mediating role of dynamic managerial capabilities: The interplay between dominant logic and small- and medium-sized enterprises performance in China. Journal of Public Affairs, v. 20, n. 2, e2002. https://doi.org/10.1002/pa.2002

Larsson, C.; Syberfeldt, A.; Säfsten, K. (2017). How to visualize performance measures in a manufacturing SME. Measuring Business Excellence, v. 21, v. 4, 337-350. https://doi.org/10.1108/MBE-03-2017-0002

Lin, H. T. (2010). Personnel selection using analytic network process and fuzzy data envelopment analysis approaches. Computers \& Industrial Engineering, v. 59, n. 4, 937-944. https://doi.org/10.1016/i.cie.2010.09.004

Lizarzaburu, E. R. (2014). Análisis del Modelo Z de Altman en el mercado peruano. Revista Universidad y Empresa, v. 16, n. 26, 141-158. https://doi.org/10.12804/rev.univ.empresa.26.2014.05 
Ludovic, V. (1995). Contextualizar lo descontextualizado. Una necesidad del discurso gerencial. Fondo Editorial Tropykos.

Lüttich, M.; Fiege, R. (2005). Ilmenauer Beiträge zur Wirtschaftsinformatik V. Nissen und D. Stelzer Volker Nissen Die Fuzzy Balanced Scorecard. URL

Maldonado-Pinto, J. E.; Portilla-Barco, L. F. (2020). Procesos de innovación en la industria manufacturera colombiana. Revista CEA, v. 6, n. 11, 145-163. https://doi.org/10.22430/24223182.1395

Mallo, P. E.; Artola, M. A.; Galante, M. J.; Pascual, M. E.; Morettini, M.; Busetto, A. R. (2006). Aplicación de herramientas borrosas al balance scorecard. Costos y Gestión, v. 16, n. 61, 12-19.

Mamdani, E. H.; Assilian, S. (1975). An Experiment in Linguistic Synthesis with a Fuzzy Logic Controller. International Journal of Man-Machine Studies. v. 7, n. 1, 1-13. https://doi.org/10.1016/S0020-7373(75)80002-2

Martínez Rojas, N.; Andrade Sosa, H. H. (2016). Integración de la lógica difusa a la dinámica de sistemas para la selección de terrenos de cultivos agrícolas. Revista Elementos, v. 6, n. 6, 149166. URL

Medina, J. (2007) El Modelo Integral de Productividad, Bogotá, Fondo de Publicaciones Universidad Sergio Arboleda.

Medina Fernandez de Soto, J. E. (2009). Modelo Integral para Optimizar la Productividad. Seventh LACCEI Latin American and Caribbean Conference for Engineering and Technology (LACCEI'2009). URL

Merigó, J. M.; Peris-Ortiz, M. (2014). Entrepreneurship and Decision- Making in Latin America. Innovar, v. 24, n. 1, 101-111. http://dx.doi.org/10.15446/innovar.v24n1spe.47557

Mintzberg, H.; Raisinghani, D.; Theoret, A. (1976). The Structure of "Unstructured" Decision Processes. Administrative Science Quarterly, v. 21, 246-275. https://doi.org/10.2307/2392045

Murnighan, J. K.; Mowen, J. C. (2002). The Art of High-Stakes Decision-Making: Tough Calls in a SpeedDriven World. John Wiley \& Sons.

Moody, P. E. (1991). Toma de decisiones gerenciales. Mc Graw Hill Latinoamericana.

Morcillo Loro, V. (2017). La acción educativa en el aula desde planteamientos innovadores: un estudio intensivo. Investigación en la Escuela, n. 92, 1-18. URL

Nagy, B.; Basbous, R.; Tajti, T. (2019). Lazy evaluations in Łukasiewicz type fuzzy logic. Fuzzy Sets and Systems, v. 376, n. 1, 127-151. https://doi.org/10.1016/i.fss.2018.11.014 
Nasir, H.; Haas, C. T.; Caldas, C. H.; Goodrum, P. M. (2016). An Integrated Productivity-Practices Implementation Index for Planning the Execution of Infrastructure Projects. Journal of Infrastructure Systems, v. 22, n. 2. https://doi.org/10.1061/(ASCE)IS.1943-555X.0000275

Nutt, P. C. (1993). The Formulation Processes and Tactics Used in Organizational Decision Making. Organization Science, v. 4, n. 2, 226-251. https://doi.org/10.1287/orsc.4.2.226

Organización para la Cooperación y el Desarrollo Económico OCDE. (2001). Measurement of aggregate and Industry-level productivity growth. $\underline{U R L}$

Ortega Gutiérrez, J.; Martínez Gil, J.; Valencia Botero J. C. (2010). El modelo de calificación crediticia Z-Score: aplicación en la evaluación del riesgo crediticio de HB Fuller Colombia Ltda. Revista MBA EAFIT, n. 1, 102-111. URL

Özdaban, I.; Özkan, C. (2011). A case study on evaluating personnel and jobs jointly with fuzzy distance sets. International Journal of Industrial Engineering, v. 18, n. 4, 169-179. URL

Özemre, M.; Kabadurmus, O. (2020), A big data analytics based methodology for strategic decision making. Journal of Enterprise Information Management. https://doi.org/10.1108/JEIM-08-2019-0222

Pérez Rave, J.; La Rotta, D.; Sánchez, K.; Madera, Y.; Restrepo, G.; Rodríguez, M.; Vanegas, J.; Parra, C. (2011). Identificación y caracterización de mudas en Pymes manufacureras incorporando la perspectiva del nivel operativo. Ingeniare. Revista chilena de ingeniería, v. 19, n. 3, 396-408. https://doi.org/10.4067/S0718-33052011000300009

Pérez Pueyo, Á. (2016). El Estilo Actitudinal en Educación Física: Evolución en los últimos 20 años. Retos, n. 29, 207-215. URL

Prince, S. E.; Vera, E. S. (2005). La indeterminabilidad en la toma de decisiones. En IX Congreso de Ingeniería de Organización. $\underline{U R L}$

Quintero Garzón, M. L.; Sánchez Fernández, M. D. (2018). Responsabilidad social corporativa: una mirada integral en América Latina. URL

Rivas, L. A. (2009). Evolución de la teoría de la organización. Revista Universidad \& Empresa, v. 7, 1132. $\underline{U R L}$

Rodríguez Cruz, Y. (2014). Modelo de uso de información para la toma de decisiones estratégicas en organizaciones de información cubanas (Tesis doctoral). Universidad de Granada, Granada, España.

Rodríguez Cruz, Y.; Pinto Molina, M. (2010). Evolución, particularidades y carácter informacional de la toma de decisiones organizacionales. Revista Cubana de Información en Ciencias de la Salud, v. 21, n. 1, 57-77. URL 
Ross, T. (2004). Fuzzy Logic with Engineering Applications. John Wilwy and Sons.

Ruvalcaba Coyaso, F. J.; Vermonden, A. (2015). Lógica difusa para la toma de decisiones y la selección de personal. Universidad \& Empresa, v. 17, n. 29, 239-256. https://dx.doi.org/10.12804/rev.univ.empresa.29.2015.10

Saaty, T. L.; Peniwati, K.; Shang, J. S. (2007). The analytic hierarchy process and human resource allocation: Half the story. Mathematical and Computer Modelling, v. 46, n. 7-8, 1041-1053. https://doi.org/10.1016/i.mcm.2007.03.010

Saaty, T. L.; Vargas, L. G. (2012) Models, methods, concepts \& applications of the analytic hierarchy process. Springer.

Shull, F. A.; Delbecq, A. L.; Cummings, L. L. (1970). Organizational decision making. McGraw-Hill, Inc.

Simon, H. A. (1997). Administrative Behavior. The Free Press.

Strefezza, M. (2009). Lógica difusa, un punto de vista. Ciencia e Ingeniería, v. 30, n. 3, 259-268. URL

Sugeno, M. (1985). Industrial Applications of Fuzzy Control. Elsevier Science Pub. Co.

Summanth, D. (1990). Administración de la Productividad Total. Mc Graw Hill.

Thornton, G. C.; Gibbons, A. M. (2009). Validity of assessment centers for personnel selection. Human Resource Management Review, v. 19, n. 3, 169-187. https://doi.org/10.1016/j.hrmr.2009.02.002

Tschang, T. (2003). China's software industry and its implications for India. URL

Valenzuela, J. (2008). Habilidades de pensamiento y aprendizaje profundo. Revista Iberoamericana de Educación, v. 46, n. 7, 1-9. https://doi.org/10.35362/rie4671914

Vélez-Salazar, F. M.; Duque-Osorio, G. A. (2017). Gestión de una comunidad de práctica. Caso de estudio: incidentes de infraestructura de dos pymes de la ciudad de Medellín. Revista CEA, v. 3, n. 5, 59-68. https://doi.org/10.22430/24223182.649

Yung, K. L.; Ko, S. M.; Kwan, F. Y.; Tam, H. K.; Lam, C. W.; Ng, H. P.; Lau, K. S. (2006). Application of Function Deployment Model in Decision Making for New Product Development. Concurrent Engineering, v. 14, n. 3, 257-267. https://doi.org/10.1177/1063293X06068392

Zadeh, L. A. (1965). Fuzzy sets. Information and Control, v. 8, n. 3, 338-353. https://doi.org/10.1016/S0019-9958(65)90241-X

Zadeh, L. A. (1968). Communication Fuzzy Algorithms. Information and control, v. 12, n. 19, 94-102. 